OPEN ACCESS

Edited by:

Zhi-Han Zhu,

Harbin University of Science and

Technology, China

Reviewed by:

Wei Gao,

Harbin University of Science and

Technology, China

Yanping Xu,

Shandong University, China

Xing $\mathrm{Fu}$,

Tsinghua University, China

*Correspondence:

Yulei Wang

wy@hebut.edu.cn

Specialty section:

This article was submitted to

Optics and Photonics,

a section of the journal

Frontiers in Physics

Received: 26 July 2021

Accepted: 30 August 2021

Published: 14 September 2021

Citation:

Cao C, Wang Y, Bai Z, Li Y, Yu Y and Lu Z (2021) Developments of Picosecond Lasers Based on Stimulated Brillouin Scattering

Pulse Compression.

Front. Phys. 9:747272.

doi: 10.3389/fphy.2021.747272

\section{Developments of Picosecond Lasers Based on Stimulated Brillouin Scattering Pulse Compression}

\author{
Chen Cao ${ }^{1,2}$, Yulei Wang ${ }^{1,2 *}$, Zhenxu Bai ${ }^{1,2,3}$, Yunfei $L^{1,2}, Y u Y u^{1,2}$ and Zhiwei $L u^{1,2}$ \\ ${ }^{1}$ Center for Advanced Laser Technology, Hebei University of Technology, Tianjin, China, ${ }^{2}$ Hebei Key Laboratory of Advanced \\ Laser Technology and Equipment, Tianjin, China, ${ }^{3}$ MQ Photonics Research Centre, Department of Physics and Astronomy, \\ Macquarie University, Sydney, NSW, Australia
}

Pulse compression based on stimulated Brillouin scattering (SBS) is a nonlinear optical approach that efficiently converts high-energy nanosecond pulses into the picosecond. Since the first observation of SBS pulse compression, different compression structures for different input and output parameters were developed to optimize the characteristics of pulse compression in the past decades. Here, a comprehensive review of the development status of SBS pulse compression schemes is provided, meanwhile, methods and trends to the optimization of SBS pulse compression are proposed.

Keywords: stimulated Brillouin scattering, pulse compression, high energy, picosecond, structure

\section{INTRODUCTION}

High-energy picosecond pulse lasers have attracted significant attention for their extensive and important applications, such as satellite laser ranging [1-3], laser processing [4-6], and medical laser treatment $[7,8]$. In the field of satellite laser ranging, ultra-high-precision remote ranging can be achieved using picosecond lasers. For example, measurement of the distance between the earth and the moon [9], the geosynchronous orbit satellite with ranging accuracy of sub-centimeter [10], and BeiDou navigation satellite system with single ranging accuracy of $8.5 \mathrm{~mm}$ [11]. For laser processing, due to the shorter pulse duration compared to that of the nanosecond, picosecond lasers can effectively inhibit the formation of heat-affected zones, realizing higher precision in processing materials. In addition, the higher peak power density can greatly improve the material removal rate and the quality of the burnt surface while the material is surface-textured [12-14]. Mode-locking is the most common approach to generate picosecond pulses, which demonstrates extremely low single pulse energy ( nJ to $\mu \mathrm{J})[15-18]$. Q-switching is another method to generate short pulses, but it is difficult to obtain pulses narrower than 300 ps due to the limitation of cavity length and switching speed [19-22]. Even though picosecond pulses can be achieved via mode-locking and Q-switching techniques, the lower power amplification efficiency induced by the short pulse duration limits their applications. Namely, the generation of picosecond pulses with high-energy directly from laser cavities and amplifiers is still a challenge.

Nonlinear optics technique provides another approach to control the temporal and spatial modes of pulses exiting a laser system. Among them, stimulated Brillouin scattering (SBS) [23], one of the strongest nonlinear light-matter interactions occurring in transparency media, has been widely studied for converting the temporal mode into a shorter one, or rather, pulse compression [24]. Today, many laser systems for high-energy picosecond pulses are realized by using pulse compression based on SBS. A key advantage of the SBS technique is generating time-reversal beam at the Stokes frequency, thus, some SBS compressors are also called SBS phase conjugate mirrors (SBS-PCMs). By appropriately using SBSPCMs, except for realizing picosecond pulses, one can also compensate wavefront distortions caused by optical elements during laser transmission and amplification processes, leading to a higher beam quality 
output $[25,26]$. For this reason, today, the combination of SBSPCMs and master oscillator power amplifier (MOPA) is a common method to achieve high peak power, high beam quality, and narrow pulse width lasers [27]. Although showed that the distortion with vortex wavefront will lead to the input "donut-like" pump pluses into peta-like Stokes pluses [28], SBS is always the most effective phase conjugation method for conventional lasers with the fundamental (i.e., $\mathrm{TEM}_{00}$ ) spatial mode.

SBS-based beam amplification technology, or named stimulated Brillouin amplification (SBA), is another effective method to obtain high-energy pulses. In this "three-wave" mixing process, the energy in the pump wave is transferred to a pre-prepared and counterpropagating Stokes wave, and parametrically generating an acoustic wave to hold the energy conservation. Compared with nonlinear crystals for optical parametric amplification (OPA), SBS media, usually are liquids, can provide a much higher limitation on the power loading. Therefore, energy of up to tens of joules can be obtained through the "beam splitting-amplification-beam combination" method [29-31]. Additionally, optical limiters, a technique for transmitting a low-intensity beam, made by SBS technology have also been widely used in high-power lasers, which can effectively protect optical elements and improve the stability of the laser system [32, 33]. Especially, based on SBS pulse compression has been demonstrated to be an effective method to convert nanosecond long pulses into picosecond short pulses due to the advantages of low cost, simple operation, and high efficiency. High-power short-pulse with peak power of $\sim \mathrm{GW}$ and pulse width of hundreds of picoseconds can be obtained through this technology [34, 35].

Besides, driven by the recent advances in the field of structured light during the last two decades, generation and application of laser beams or pulses with high-order (scalar and vector) spatial modes have gained increasing attention [36,37]. Although the study on shaping light's spatial modes via nonlinear interactions can be traced back to the dawn of the field [38], the SBS involves structured light was theoretically considered until the last decade and only explored experimentally in recent years, yet demonstrating a series of impressive results [28, 39-44]. For years, detailed research works on SBS-based pulse compression technology had been hotly pursued by several groups. Although many overviews on the research progress of SBS media have been reviewed, there are few reports on summarizing the structures of SBS pulse compressors [45, 46]. In this paper, recent research progress using different SBS pulse compression structures for picosecond pulse generation were reviewed. Meanwhile, both the advantages and disadvantages of different structures were compared and analyzed. Finally, the optimized pulse compression structure that can be applied in the future was proposed, which provided a reference for the development of a high-energy laser.

\section{PRINCIPLE OF PULSE COMPRESSION TECHNOLOGY BASED ON STIMULATED BRILLOUIN SCATTERING}

SBS is a physical process of three-wave coupling formed by the interaction of strong light and matter. The physical process of SBS is depicted in Figure 1A. The backward propagating Stokes beam is generated by the interaction of the high-intensity pump and the medium under the periodically changing acoustic grating. Then the Stokes is continuously amplified with time by the scattering effect of the acoustic grating on the pump. The process shows the characteristics of high-efficient pulse compression by the energy transfer from the pump to Stokes directly with the peak power density greatly enhanced. However, it should be noted that the efficiency of the energy transfer process (pulse compression process) will be affected by many factors, for example, the interaction length of the pump and Stokes beams. Too short interaction length will lead to low pulse compression efficiency and low energy conversion efficiency. However, too long interaction length can also result in negative effects including tail modulation and pulse width broadening. Therefore, choosing an appropriate interaction length is critical to realize highefficiency SBS pulse compression with high quality. The electrostriction effect produced by the polarization of the molecules in the medium causes the change of refractive index or density. Then, pump light is scattered by periodically changing density fluctuations which are also called moving gratings and its transmission direction is changed, enhancing the intensity of backward propagating Stokes light.

The diagram of the SBS-based pulse compression is shown in Figure 1B. The leading edge of the backward-propagating Stokes light meets the forward-propagating pumping light firstly. The energy of the pump light is transferred to the Stokes light through the phonon field vibrated in the three-wave coupling process. At this moment, the leading edge of Stokes light is magnified and the trailing edge remains unchanged. Because the energy of the pump light has almost been exhausted by the leading edge of Stokes light, and it has almost no amplification ability when it meets the trailing edge. This process is manifested as the steepening of the leading edge of the Stokes light waveform and the narrowing of the overall pulse width of the laser pulse, which is the so-called pulse compression technology. A large number of research groups have carried out exploration works for pulse compressors based on stimulated Raman scattering (SRS) and SBS [47-61]. Maier et al. and Chiao et al. successfully observed backward Raman scattering caused by forwarding Stokes radiation scattering and Brillouin scattering, which was based on noise [47, 61]. In 1972, Stolen et al. achieved the SRS-based pulse compression in glass optical waveguide for the first time [53].

\section{REASERCH PROGRESS OF STIMULATED BRILLOUIN SCATTERING PLUSE COMPRESSORS \\ Tapered Waveguide}

In the early stage, most of the pulse compression based on SBS was fulfilled in tapered waveguides. In 1980, Hon et al. for the first time realized the SBS pulse compression in a tapered waveguide filled with methane at a pressure of 130 atm [60]. Figure 2 illustrates the basic structure of the tapered waveguide pulse compressor [62]. In such a tapered fiber, the Stokes light is 

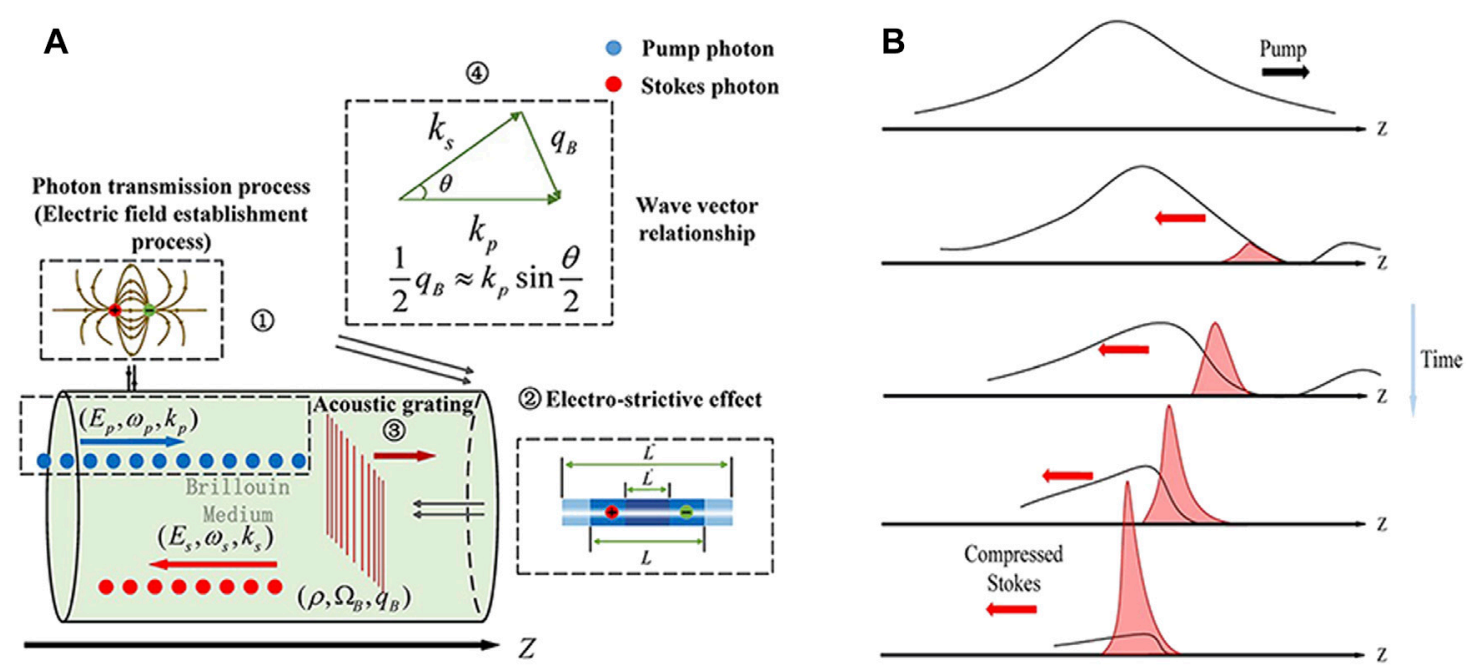

FIGURE 1 | (A) The physical process of SBS, (B) Principle diagram of pulse compression based on SBS.
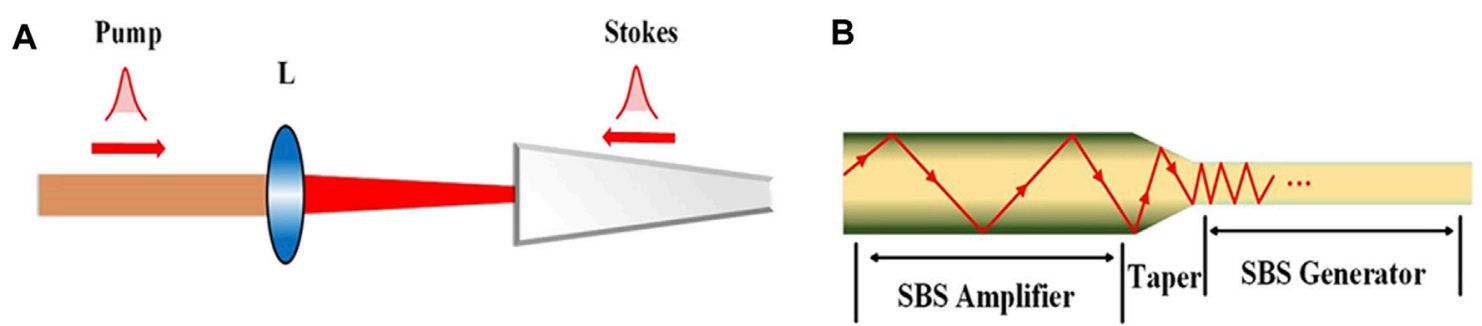

FIGURE 2 | Schematic diagram of tapered waveguide. (A) Tapered waveguide pulse compressor, (B) Schematic diagram of tapered waveguide structure [62].

first generated in the smaller core of the generator section and the main power reflection takes place in the amplifier. With this specific structure, the SBS gain is enhanced due to the smaller diameter of the optical fiber in the SBS generator. During the tapering process, both the length and angle of the taper can be controlled to obtain optimal results for a given system. For instance, a longer tapered region can effectively decrease the power coupling loss between the generator and the amplifier [63]. However, although this structure has a lower SBS threshold, it cannot be applied in SBS pulse compression experiments with high energy peak power due to the limitations of fiber material [127]. Therefore, more universal structures are required for pulse compression.

\section{Focusing Single Cell}

Compared with tapered waveguide, lens focusing structure has advantages of high stability and lower cost. In the 1980s, the focusing single-cell structure was first proposed by Damzen et al., and the basic structure was shown in Figure 3. They obtained the compressed pulse with a width of $4 \mathrm{~ns}$ in methane [64]. Since then, a large number of groups have used the structure to perform compression in different media [65-73] (as shown in Table 1). For instance, Kmetik et al. compressed the pulse width from 10 to

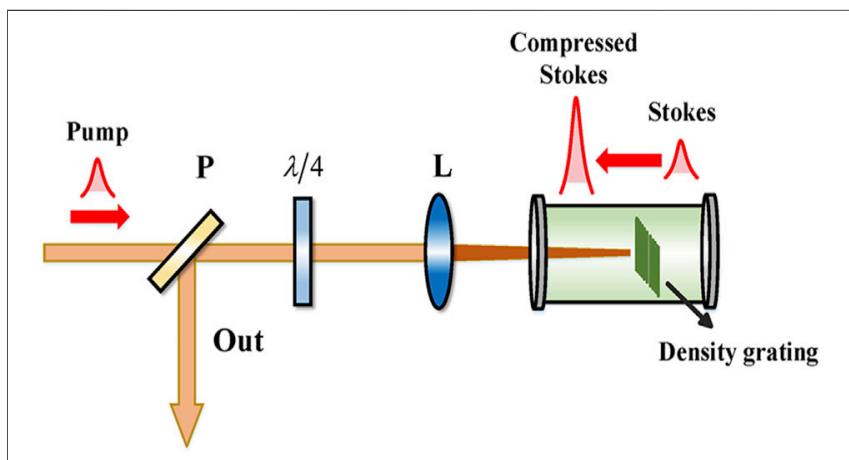

FIGURE 3 | Schematic diagram of focusing single cell structure.

$0.9 \mathrm{~ns}$ with an energy of $0.57 \mathrm{~J}$ in ultra-filtered FC-75 liquid medium and obtained up to $94 \%$ energy reflectivity [71]. Yoshida et al. proved for the first time that a $1,064 \mathrm{~nm}$ pump pulse and its second harmonic $(532 \mathrm{~nm})$, third harmonic $(335 \mathrm{~nm})$, and fourth harmonic $(266 \mathrm{~nm})$ could be compressed to hundreds of picoseconds based on a single-cell structure that demonstrated over $80 \%$ energy $[72,73]$. The above two experimental results prove that due to the advantages of 
TABLE 1 | Typical experimental results in single cell structure.

\begin{tabular}{|c|c|c|c|c|c|}
\hline Author (Year) & Medium & $\begin{array}{l}\text { Pump pulse } \\
\text { width/ns }\end{array}$ & $\begin{array}{c}\text { Compressed pulse } \\
\text { width/ns }\end{array}$ & Output energy/mJ & Energy efficiency \\
\hline Damzen et al. (1983) [62] & Methane & 27 & 4 & 10 & $70 \%$ \\
\hline Gorbunov et al. (1983) [64] & $\operatorname{Ar}$ & 20 & 1 & 500 & $80 \%$ \\
\hline Tomov et al. (1985) [65] & Freon-12 & 25 & 1.5 & 180 & $35 \%$ \\
\hline Buzyalis et al. (1985) [67] & Carbon tetrachloride & 12 & 0.7 & 1 & 1 \\
\hline Davydov et al. (1986) [68] & Carbon tetrachloride & 30 & 1.2 & 40 & $90 \%$ \\
\hline Kmetik et al. (1998) [70] & FC-75 & 10 & 0.9 & 570 & $94 \%$ \\
\hline Yoshida et al. $(2004,2007)[71,72]$ & Fused Silica & 8 & 1 & 1,000 & $40 \%, 95 \%$ \\
\hline
\end{tabular}

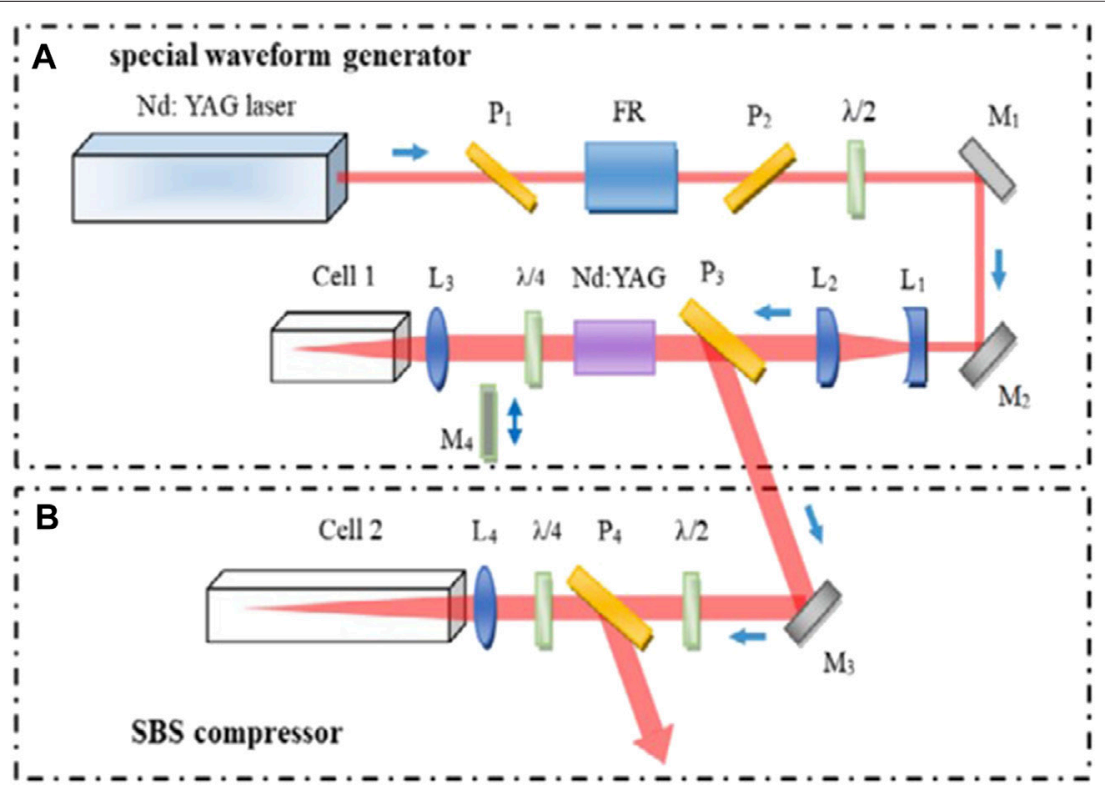

FIGURE 4 | Schematic diagram of experimental setup: (A) special waveform generator, (B) single-pass SBS compressor [74].

compact structure and easy adjustment, using the focusing single cell is easy to obtain high energy reflectivity. In 2016, Liu et al. conducted comparative experiments on pulse compression of pump light with different waveforms, and the results showed that the pulse width of the step pulse could be compressed to half of the phonon lifetime with a single-cell with energy efficiency exceeding $65 \%$. The schematic diagram of the experimental device is shown in Figure 4 [74]. Bai et al. developed a picosecond laser with $<450 \mathrm{ps}$ pulse width and single pulse energy of $400 \mathrm{~mJ}$ by combining MOPA with SBS pulse compression [75]. In 2018, Liu et al. applied a single-cell structure based on an interferometric scheme to break through the pulse compression limit (the phonon lifetime of the medium). They obtained a compressed pulse output of $0.36 \tau_{B}$ with energy efficiency of $65 \%$ in FC-3283, and a compressed pulse output of $0.12 \tau_{B}$ with energy efficiency of $40 \%$ in Acetone, which was close to one-tenth of the phonon lifetime [76].

The focusing single-cell structures have the advantages of simple structure and easy operation. However, with the increasing of injected energy, the low load capacity of this structure will lead to the phenomena of optical breakdown, hindering the generation of Stokes seed light in severe cases. Therefore, the single-cell structure is mostly used in small and low-energy commercial laser systems. Piskarskas et al. theoretically analyzed the space-time characteristics of the Stokes beam formed when the pump light intensity was unevenly distributed in spatial cross-sectional. They proposed that the two-cell generator-amplifier setup should be preferred to realize a compression ratio higher than 10 [77].

\section{Focusing Two-Cell Structure}

To overcome the shortcomings of the focusing single-cell structure, the two-cell structure was introduced to separate the compression part and the generating part of the original single cell into two independent cells. The design enables the control of the energy which is injected into the generator, thereby improving the load capacity and stability of the entire system. In addition, the interaction length between pump light and Stokes seed light is increased, which is beneficial to obtain a high compression ratio. 


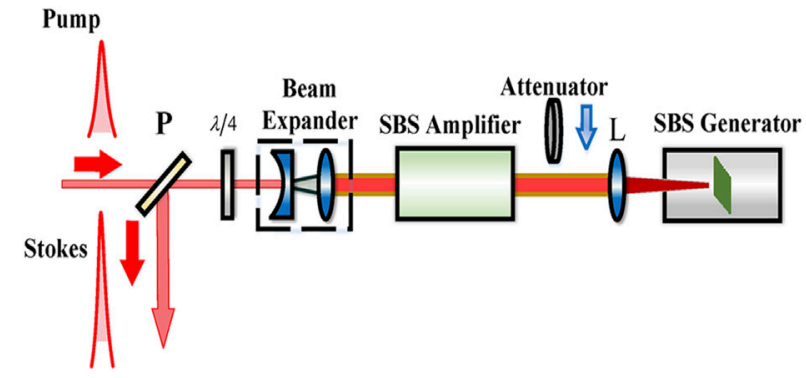

FIGURE 5 | Schematic diagram of compact two-cell structure.

Compact two-cell and independent two-cell are the two most used structures.

\section{Compact Two-Cell}

Figure 5 illustrates the schematic diagram of a compact two-cell structure. In general, an attenuator is placed in front of the focusing lens to reduce the energy entering the generator, or a beam expander system is placed in front of the amplifier to reduce the intensity of the pump pulse. This is to avoid irreversible damage to components caused by optical breakdown. Schiemann et al. conducted compression experiments in three liquid media: water, methanol, and carbon tetrachloride. The minimum pulse width of 270 ps (central part of the beam) was obtained with a reflectivity of $75 \%$ [78].

In recent years, great breakthroughs have been made in pulse compression ratio and energy efficiency [79-89] (as shown in Table 2). Yoshida et al. compressed the pulse width from 13 to 160 ps with a central wavelength of $1,064 \mathrm{~nm}$ in an FC-40 liquid medium. The beam brightness was increased by nearly 65 times, and the highest energy efficiency exceeded $80 \%$ without optical breakdown (the experimental setup is shown in Figure 6) [84]. Wang et al. proposed a new type of cyclic compact two-cell structure for realizing SBS-PCM with a high-energy and highrepetition rate. Energy reflectivity of $84.7 \%$ was obtained with an incident energy of $1.1 \mathrm{~J}$ at a repetition rate of $10 \mathrm{~Hz}$ [79]. Hasi et al. compressed the $10 \mathrm{~ns}$ to $116 \mathrm{ps}$ in different perfluorocarbon liquid media, generating the single pulse energy over several hundred millijoules with energy reflectivity over $80 \%[80,81,85]$.

In 2015, Wang et al. proposed an interferometric scheme to generate standing waves in the focal area to improve energy stability. The pulse was compressed from $8 \mathrm{~ns}$ to 393 ps with a single pulse energy of $40 \mathrm{~mJ}$ with high stability [87, 88]. In 2017 ,
Bai et al. achieved picosecond ultraviolet ( $355 \mathrm{~nm}$ ) output with compact two-cell structure. The pulse width was compressed from $6.3 \mathrm{~ns}$ to $168 \mathrm{ps}$ with an output energy of $100 \mathrm{~mJ}$ and the energy efficiency of about 35\% [89]. In 2019, Wang et al. obtained a short pulse of $820 \mathrm{ps}$ at $\mathrm{kHz}$ repetition rate in HT270 liquid medium with energy efficiency of $52.2 \%$ for the first time [90].

Due to the advantages of higher load capacity and longer effective interaction length, the compact two-cell structure can obtain shorter pulse width and higher energy efficiency with different SBS active media. But the energy stability becomes worse with the increase of pump energy, which is similar to the focusing single cell structure. Controlling energy through the attenuator also has several defects, such as low energy efficiency and adjustment accuracy. In 2006, Mitra et al. proposed a new type of "one-cell, dual-purpose" compact two-cell structure [91]. Their results show that more than $90 \%$ energy efficiency can be obtained with the pulse width close to $1 \mathrm{~ns}$ at the injected energy reaching above $1-\mathrm{J}$ level without inserting an attenuator. The minimum pulse duration of 600 ps could be obtained when the attenuation was $45 \%$, while the energy efficiency was only $55 \%$. Kmetik et al. designed the compact dual-cell compressor which was applied to the GEKKO-XII laser device, realizing highenergy pulse compression with a large-diameter laser system [92]. The pulse was compressed from $13 \mathrm{~ns}$ to $600 \mathrm{ps}$ at the output energy up to $30 \mathrm{~J}$ approximately with the low energy efficiency. Therefore, the compact two-cell structure is unsuitable to be applied in the situation of high energy. At this time, an independent two-cell structure was proposed which could flexibly adjust the injected energy of two-cell, respectively.

\section{Independent Two-Cell}

The independent two-cell structure is actually a kind of light splitting structure, which divides the pump light into two parts: seeding pump light and amplifying pump light (as shown in Figure 7). The utilizing of optical elements such as waveplates and polarizers can flexibly control the meeting position, meeting time, and intensity ratio of the two beams. The structure effectively improves the load capacity of the entire system under high energy. In 1984, Fedosejevs et al. first proposed an independent two-cell structure and using $\mathrm{KrF}$ laser to compress long pulses from $24 \mathrm{~ns}$ to 440 ps with the energy efficiency of about $40 \%$ [93, 94].

Compared with other structures, the obvious advantage of the independent two-cell structure is adjusting the pulse intensity in the two cells arbitrarily, resulting in avoiding nonlinear effects such as an optical breakdown. In the follow-up research, more detailed research and optimization of the optical path structure

TABLE 2 | Typical experiments of compact two-cell structure.

\begin{tabular}{|c|c|c|c|c|c|}
\hline Author (Year) & Medium & $\begin{array}{l}\text { Pump pulse } \\
\text { width/ns }\end{array}$ & $\begin{array}{l}\text { Compressed pulse } \\
\text { width/ns }\end{array}$ & Output energy/mJ & $\begin{array}{c}\text { Energy efficiency } \\
(\%)\end{array}$ \\
\hline I.Daito et al. (2012) [79] & FC-40 & 8 & 0.2 & 300 & 80 \\
\hline Hasi W et al. (2013) [80] & FC-770 & 8 & 0.116 & 260 & 80 \\
\hline Zhu et al. (2014) [81] & FC-40 & 8 & 0.136 & 300 & 80 \\
\hline Ogino et al. (2014) [82] & FC-40 & 4 & 0.4 & 34 & 75 \\
\hline
\end{tabular}


TABLE 3 | Summary of the advantages and disadvantages of each structure.

\begin{tabular}{lll}
\hline Name of structure & \multicolumn{1}{c}{ Advantage } & Disadvantage \\
\hline Tapered waveguide & Simple structure, easy to control & High cost, poor universality \\
Focusing single cell & Simple optic path, more controllable compression & Poor stability under high-energy condition \\
Compact two-cell & Longer effective interaction length, can obtain higher compression ratio & Poor stability under high-energy condition \\
Independent two-cell & Strong flexibility & Complicated optical path, large loss \\
Non-focusing structure & Strong stability, suitable for high-power large-aperture laser system & There are pump source modulation problems and threshold problems
\end{tabular}

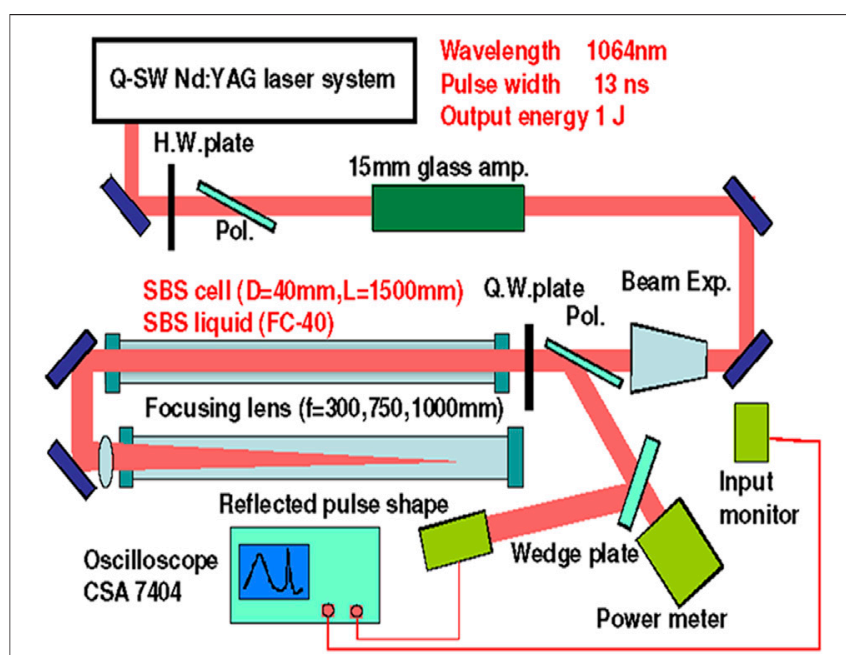

FIGURE 6 | Schematic diagram of typical experiment design of compact two-cell [84].

were carried out to achieve more precise and stable control of the beam intensity [128].

Dane et al. added an adjustable delay light path for changing the relative delay time of the seeding pump light and the amplifying pump light in the independent two-cell structure for the first time. The scheme obtained output energy of $2.5 \mathrm{~J}$, and proposed a new optimization direction for compression characteristics [95]. From 2014 to 2017, Xu et al. conducted a series of pulse compression experiments. Compressed pulse width from $12 \mathrm{~ns}$ to $580 \mathrm{ps}$ at the center wavelength of $1,064 \mathrm{~nm}$ in FC-72 was performed. At the center wavelength of $532 \mathrm{~nm}$ in water, the pulse width was compressed from $9 \mathrm{~ns}$ to 170 ps which was close to the phonon life limit of the medium. At the same time, they explored the relationship between the meeting position of the two light and the magnification effect in the independent two cell structure. In the two experiments, the energy reached $1 \mathrm{~J}$ level with the peak power over $1 \mathrm{GW}$ [97-99, 129, 130].

The independent two-cell structure can largely avoid the nonlinear effects such as optical breakdown, self-focusing, and selfdefocusing under high-energy conditions, which inclines it to obtain better compression output characteristics and beam quality. However, introduced additional optical elements directly cause considerable transmission energy losses. Consequently, it is unfavorable for obtaining high energy efficiency. At the same time, the complexity of the optical path and the adjustment difficulty cannot be ignored, especially in the application of large-scale laser systems.

\section{Other Structures}

\section{Multi-Stage Compression Structure}

The multi-stage compression method provides the possibility to compress the pulse width to below 100 ps [100-108]. From 2003 to 2006, Wang et al. compressed the pulse width from $8 \mathrm{~ns}$ to 153 ps with two-stage compression in FC-75 [102, 103]. Pivinskii et al. proposed a new three-stage pulse compression structure for the first time. It was composed of a two-stage SBS compression system and a terminal SRS compression system. The original pump pulse of $8 \mathrm{~ns}$ was compressed to the ultrashort pulse of $20 \mathrm{ps}$ in carbon tetrachloride liquid with an output energy of $6 \mathrm{~mJ}$. At the same time, it was proposed that pulses below $10 \mathrm{ps}$ could be obtained with lower output energy [106]. Figure 8 illustrates the schematic diagram of the two-stage compression.

Generally, large-aperture laser systems rely heavily on beam quality. Although the multi-stage compression structure has made a great breakthrough with pulse width, the problems such as wavefront distortion and poor stability caused by the increasing of components have seriously affected the beam quality and energy efficiency. Therefore, improving the beam quality is the core issue for this structure to be applied in largeaperture high-power laser systems in the future.

\section{Non-focusing Structure}

For a long time, reports on the structure of SBS pulse compressor have basically focused on the lens focusing structure [72, 81, 98, 109]. To improve the stability under high-energy conditions, Neshev et al. proposed a new non-focusing single-cell structure for the first time and compressed the pulse width from $4 \mathrm{~ns}$ to 200 ps [108]. In 2015, our group obtained a short pulse of $\sim 360$ ps with a single pulse energy of $3.02 \mathrm{~J}$, and there was no thermal effect or other nonlinear effects during the compression process [109]. Figure 9 provides a schematic diagram of the experimental device and principle of the non-fousing method. The high reflectivity mirror was used to reflect the Stokes component of the sideband component of the super-Gaussian pump light to form the "feedback-initiated" Stokes seed light, and then compressing and amplifying. This method can suppress the generation of seed light originating from randomly distributed thermal noise. Thereby it reduces the probability of phase distortion and improves the stability of the system and the beam quality of the output pulse. Although this structure can 


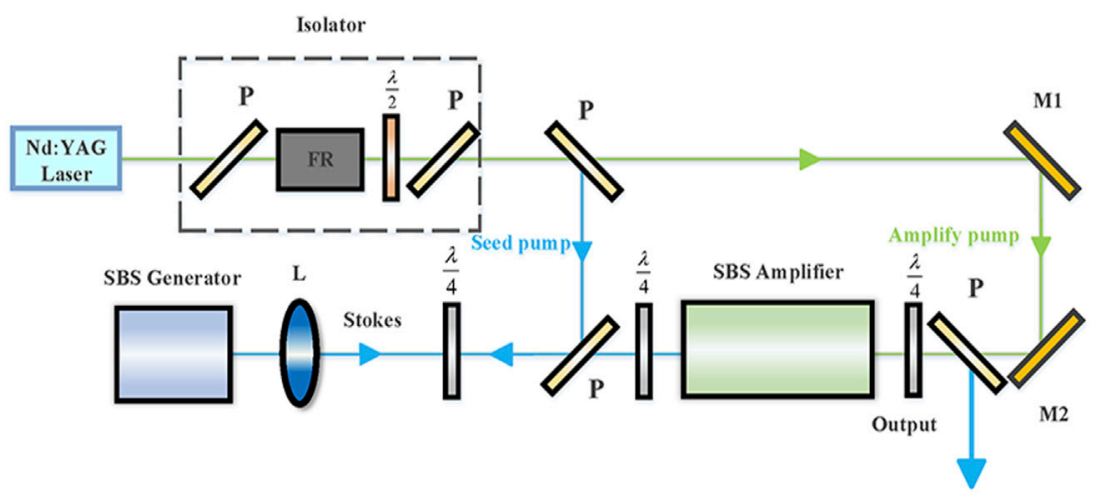

FIGURE 7 | Schematic diagram of basic independent two-cell structure.

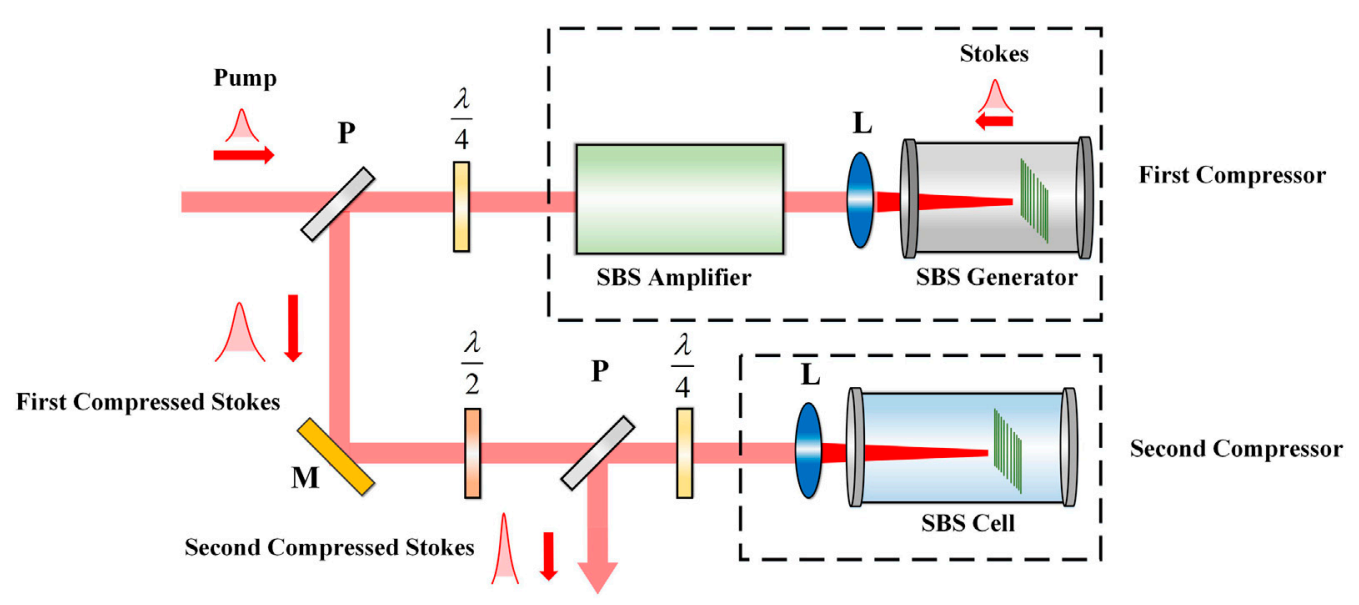

FIGURE 8 | Schematic of the two-stage compression structure.

be used for high-energy pulse compression characteristics research experiments, its injection energy will be limited by the noise-initiated SBS threshold in the medium cell. In addition to the mentioned challenges, the modulation procedure of the pump pulse cannot be removed because of the non-conjugation in this process. Table 3 lists the advantage and disadvantages of different structures.

\section{Unfolding the Hidden Spatial Degree of Freedoms \\ Plasma-Based Stimulated Brillouin Scattering}

In recent years, some research groups use plasma as the new SBS active medium for pulse compression and amplification. Due to the advantage of its non-destructive property, SBS pulse compression based on plasma can obtain extremely high peak power up to the order of $10^{15} \mathrm{~W}$ and ultrashort pulse of the order of femtosecond [96, 110-114]. In 2010, Lancia et al. achieved the energy transfer from a long ( $3.5 \mathrm{ps}$ ) pump pulse to a short (400 fs) seed pulse due to stimulated Brillouin backscattering in the strong-coupling regime with output energy of $60 \mathrm{~mJ}$ in $\mathrm{N}_{2}$ and
Ar plasmas. The interaction process is shown in the Figure 10. They found that increasing the uniformity of plasma density can effectively reduce energy attenuation. At the same time, maintaining a high plasma density can increase the maximum amplification factor [96]. In 2013, Weber et al. used the strong coupling stimulated Brillouin technology in the plasma to obtain an amplified seed light with a peak power of $10^{18} \mathrm{~W} / \mathrm{cm}^{2}$ and a pulse width of $10 \mathrm{fs}$ [113].

\section{Stimulated Brillouin Scattering With Structured Light}

To date, the great majority of SBS studies, as reviewed in above, neglected the spatial dimensions of paraxial light. This is because most commercial laser systems are designed to generate the $\mathrm{TEM}_{00}$ mode with a linear polarization for highly efficient generation. More recently, progress in the study on structured light, both in fundamental and application aspects, has significantly gained the research intertest in techniques for generation and manipulation of high-order spatial modes [36, 37]. For the nonlinear approach, OAM transfer between light fields in varies optical parametric processes have been intensively studied over 20 years [38], and the community is recently focus 

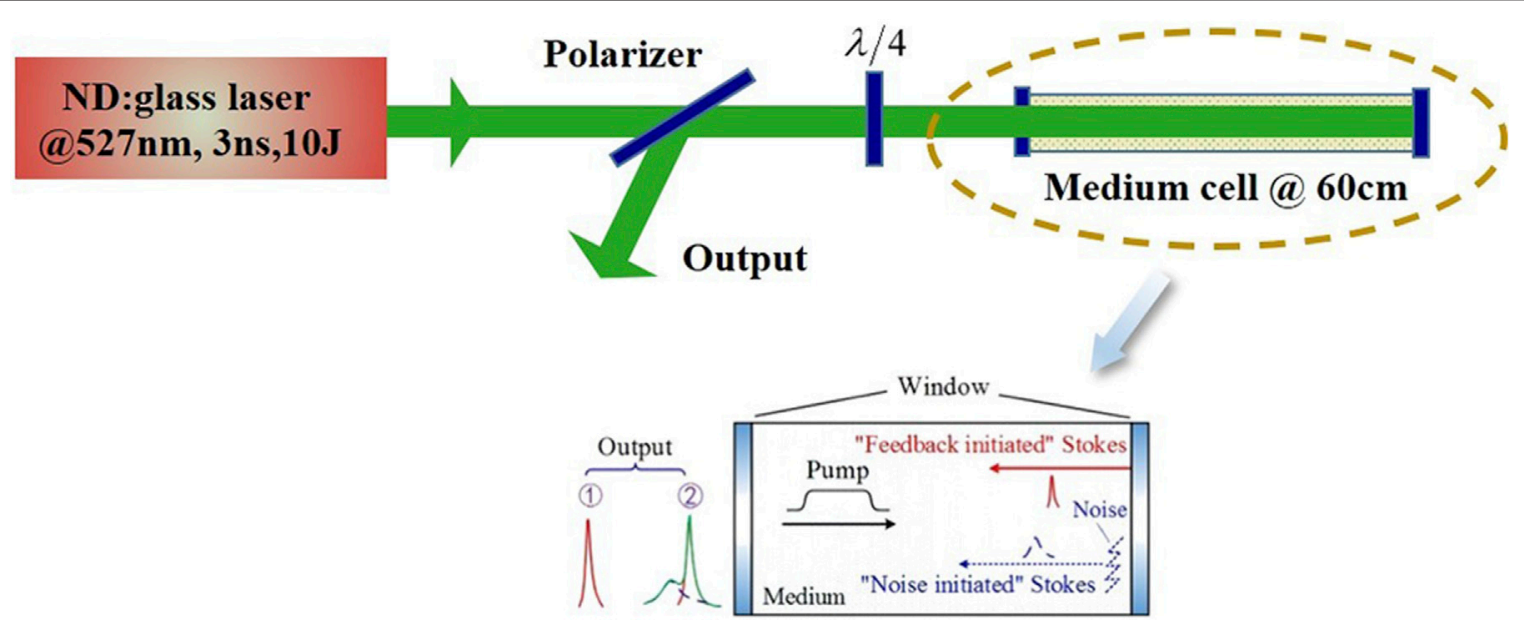

FIGURE 9 | The schematic diagram of "feedback-initiated" non-focus structure experimental device and principle: (1)represents the "feedback-initiated" Stokes reflected pulse waveform; (2) represents the coexist of "double peak" reflected pulse waveform of "noise-initiated" Stokes and "feedback-initiated" Stokes [109].

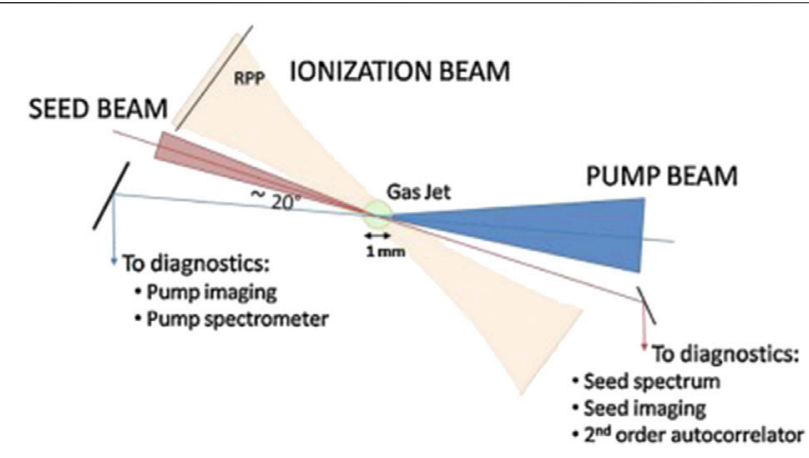

FIGURE 10 | Experimental scheme showing the three laser beams focused at the center of a supersonic gas jet [96].

on nonlinear generation and transformation generalized spatial modes and associated full-field selection rules [39, 115], as well as the effect of spin-orbit coupling [116]. By contrast, SBS involving OAM carrying light was first studied in theory until 2009 [40], and the corresponding experimental demonstration only appeared the 5 years later [41]. Particularly, Zhu et al. systematically studied OAM selection rules in varies SBS processes, and first realized OAM interconversion between light and acoustic fields, a high-dimension light memory, and revealed the underlying physics for the fragmentation of vortex beams in SBS-PCMs [28, 39], as shown in Figure 11.

For vectorial spatial modes, the first theoretically study for amplification of cylindrical vector $(\mathrm{CV})$ modes was provided by Vieira et al. [42], only 1 year later, Zhu experimentally realized a strong (100 mJ at $300 \mathrm{ps}) \mathrm{CV}$-mode laser output via SBA [43]. Besides, it is worthy to note that the structured acoustic wave generated in SBS excited by structured light, owing to its 0 -spin and low group velocity, has a great potential for generation and transformation of structured light, and especially for applications regarding to strong laser pulses. For instance, ref. 120 predicted a "high OAM harmonic generation in cascading SBS/SRS on a specific acoustic wave. Remarkably, some novel nonlinear optical phenomena have been revealed in these few works, yet the underlying physics for the transformation of spatial modes in SBS has not been fully understanded to date. In a word, the SBS science with structured light is still in its infancy.

\section{SUMMARIES}

Due to the advantages of simple structure, low cost, and excellent beam quality of output pulse among others, pulse compression based on SBS has become one of the effective means to obtain picosecond pulses. This review gives a comprehensive overview of the research progress of SBS pulse compression with different structures. In addition, gain media also have a significant impact on the compression characteristics of SBS. At present, the more common Brillouin media are mainly solid media such as fused silica, K8 glass and liquid media such as perfluorocarbon series and water. Among them, the solid media has good thermal conductivity and no thermal convection effect, but its damage threshold is relatively low. As a new solid gain medium discovered in recent years, diamond crystal has attracted extensive attention because of its high Brillouin gain coefficient, high thermal conductivity, and wide spectral transmission range [117-120]. Once it breaks through the size limit, its application prospect in SBS pulse compression will be very promising. As a new type of Brillouin medium discovered in the last decades, the perfluorocarbon series of liquids have excellent performance and low cost, therefore are currently the mainstream Brillouin medium in high energy pulse compression. In general, since Stokes beam with narrow pulse width can be easily obtained, media with short phonon lifetime and high gain coefficient are popular. However, based on recent experimental results, our research group found that the medium which has a 


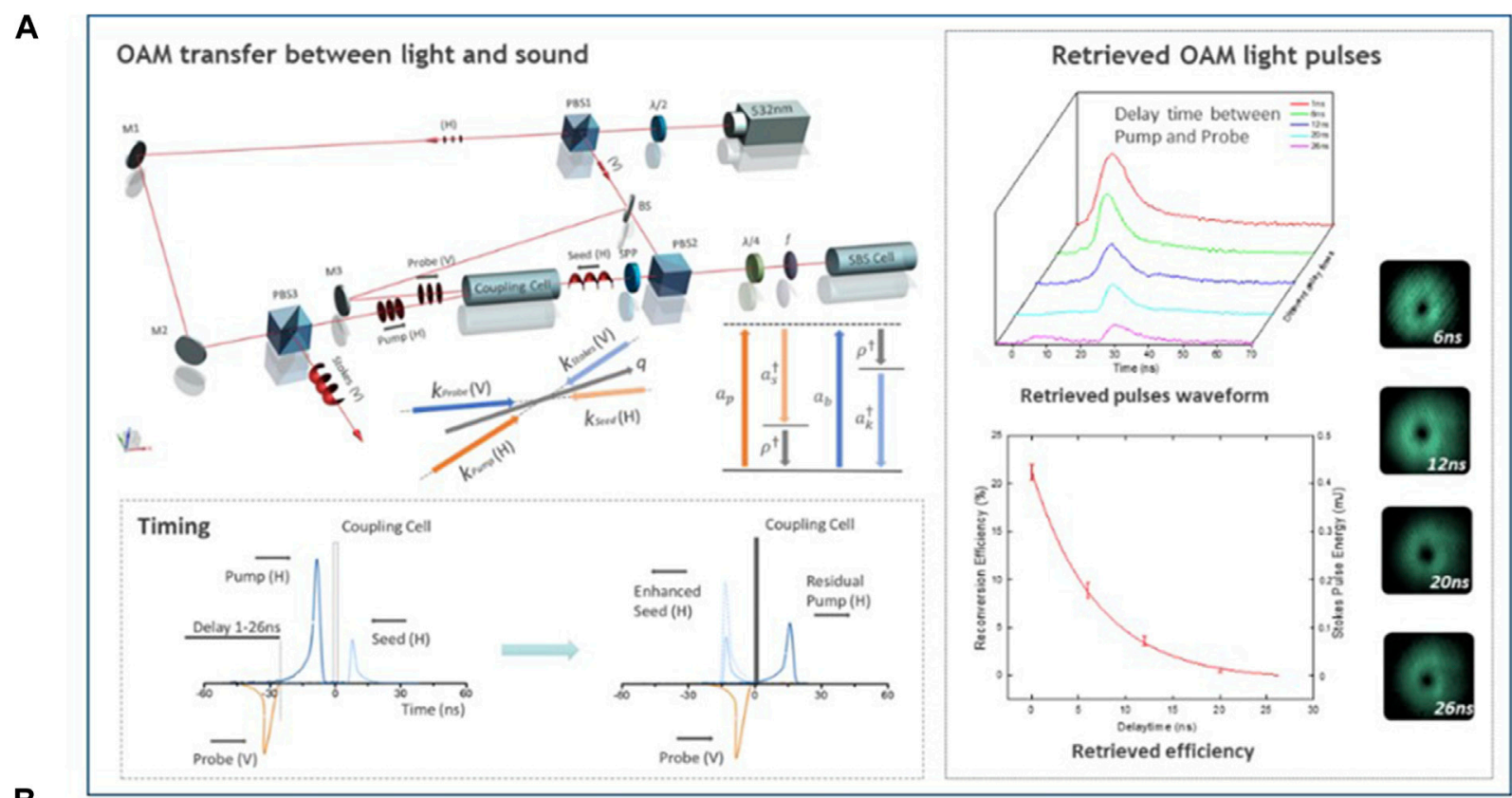

B
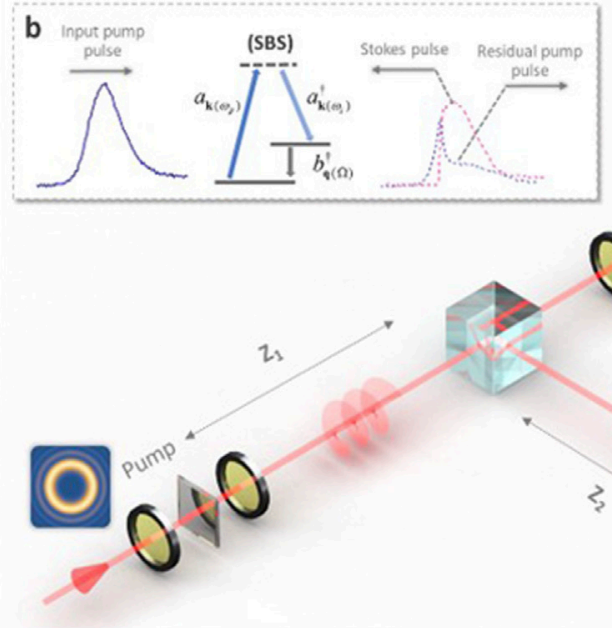
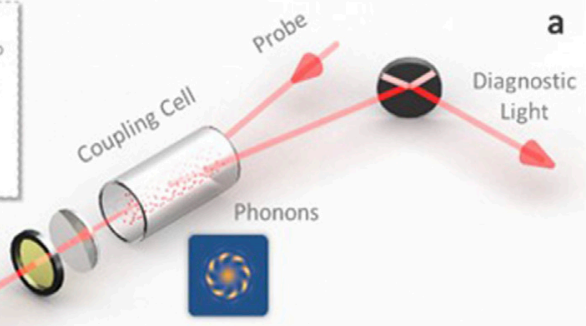

a
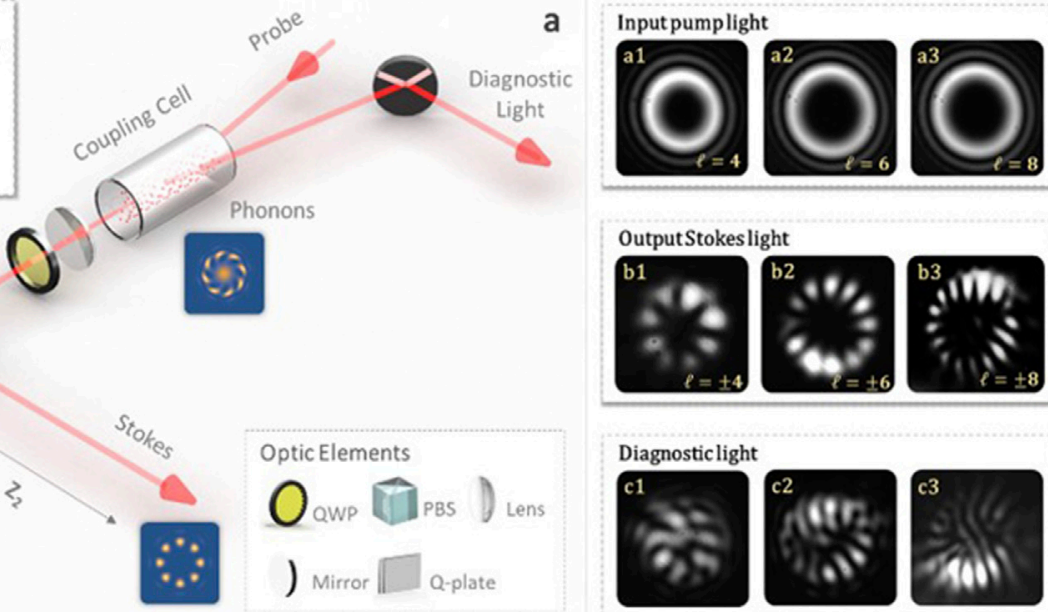

Optic Elements

OQWP 1 PBS $)_{\text {Lens }}$

) Mirror $\square$ a-plate
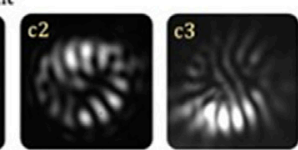

FIGURE 11 |Realization of OAM interconversion between light and sound waves via SBS (A) and demonstration of fragmentation of vortex beams in an SBS-PCM (B) $[28,39]$.

short phonon lifetime and high gain (such as HT-230) will cause the compression limit point to be generated prematurely, and then the pulse width broadening phenomenon and even the cascaded SBS phenomenon will occur.

At present, more attention to SBS-based pulse compression and amplification studies has been paid to narrower pulse width output with higher repetition rate and higher energy. Since the traditional focusing structure and active medium are limited by the damage threshold, it is difficult to make major breakthroughs with an aspect of pulse intensity. Therefore, the researches on non-focusing structures and new active media have guiding significance. The non-focusing structure can greatly improve the stability of the entire system and the injected energy, and obtain short pulses with high beam quality. It is extremely suitable for high-power large- aperture laser systems. Chirped-pulse amplification technology (CPA) and optical parametric chirped-pulse amplification technology (OPCPA) have been unable to achieve further breakthroughs because of the limitation of the optical damage threshold of compressed gratings [121-125]. At the same time, the reversibility of photoacoustic conversion in the SBS process has been reported, and it also has application value in communication [126]. Although the above-mentioned strong coupling stimulated Brillouin scattering technology implemented in plasma has obtained extremely high peak power and ultra-short pulse width, this method is hard to manipulate, and has extremely high requirements for the experimental environment and the precision of the devices. Therefore, the plasma-based SBS pulse compression technology still needs in-depth research. The 
breakthroughs of this technology will significantly promote research work and practical applications in the field of basic science.

\section{AUTHOR CONTRIBUTIONS}

CC: Writing-Original Draft, Writing-Review and Editing. YW: Supervision, Funding acquisition, Writing-Review and Editing.

\section{REFERENCES}

1. Bury G, Sośnica K, Zajdel R. Multi-GNSS Orbit Determination Using Satellite Laser Ranging. J Geod (2018) 93(12):2447-63. doi:10.1007/s00190-0181143-1

2. Loomis BD, Rachlin KE, Wiese DN, Landerer FW, Luthcke SB. Replacing GRACE/GRACE-FO with Satellite Laser Ranging: Impacts on Antarctic Ice Sheet Mass Change. Geophys Res Lett (2020) 47(3):e2019GL085488. doi:10.1029/2019GL085488

3. McGarry JF, Hoffman ED, Degnan JJ, Cheek JW, Clarke CB, Diegel IF, et al. NASA's Satellite Laser Ranging Systems for the Twenty-First Century. J Geod (2018) 93(11):2249-62. doi:10.1007/s00190-018-1191-6

4. Samanta A, Wang Q, Singh G, Shaw SK, Toor F, Ratner A, et al. Nanosecond Pulsed Laser Processing Turns Engineering Metal Alloys Antireflective and Superwicking. J Manuf Process (2020) 54:28-37. doi:10.1016/ j.jmapro.2020.02.029

5. Sugioka K. Progress in Ultrafast Laser Processing and Future Prospects. Nano-photonics (2017) 6(2):393-413. doi:10.1515/nanoph-2016-0004

6. Zheng Z, Wu C, Liu S, Yang X. Analysis of Energy Occupying Ratio of Coulomb Explosion and Thermal Effect in Picosecond Pulse Laser Processing. Opt Commun (2018) 424:190-7. doi:10.1016/ j.optcom.2018.04.045

7. Zhan SY, Li HT, Yang RY. New Progress in Laser Treatment of Chloasma. J Pract Dermatol (2018) 11(3):158-61. doi:10.11786/sypfbxzz.1674-1293.20180309

8. Li M, Huang Y, Zhu W. Clinical Application Progress of Picosecond Laser. J Pract Dermatol (2019) 12(4):223-6. doi:10.11786/sypfbxzz.16741293.20190409

9. Huang Y, Zhang H, Yan X, Guo G, Bai Z, Lin W, et al. High-Brightness $100 \mathrm{~Hz} / 363 \mathrm{~mJ}$ Picosecond Nd:YAG Laser System for Ultra-remote Laser Ranging. IEEE J Quan Electron. (2020) 56(5):1700310. doi:10.1109/ JQE.2020.3004139

10. Zhang H, Cheng Z, Long M, Deng H, Meng W, Wu Z, et al. Applications of Satellite Laser Ranging and Laser Time Transfer in BeiDou Navigation Satellite System. Optik (2019) 188:251-62. doi:10.1016/j.ijleo.2019.04.131

11. Sośnica K, Bury G, Zajdel R, Strugarek D, Drożdżewski M, Kazmierski K. Estimating Global Geodetic Parameters Using SLR Observations to Galileo, GLONASS, BeiDou, GPS, and QZSS. Earth Planets Space (2019) 71(11):20. doi:10.1186/s40623-019-1000-3

12. Benocci R, Batani D, Roman HE. Incubation Models for Under-threshold Laser Ablation with Thermal Dissipation. Appl Phys B (2019) 125(2):22. doi:10.1007/s00340-019-7132-0

13. Mustafa H, Matthews DTA, Römer GRBE. The Role of Pulse Repetition Rate on Picosecond Pulsed Laser Processing of $\mathrm{Zn}$ and $\mathrm{Zn}$-Coated Steel. Opt Laser Technol (2020) 131:106408. doi:10.1016/j.optlastec.2020.106408

14. Mustafa H, Pohl R, Bor TC, Pathiraj B, Matthews DTA, Römer GRBE. Picosecond-pulsed Laser Ablation of Zinc: Crater Morphology and Comparison of Methods to Determine Ablation Threshold. Opt Express (2018) 26(14):18664-83. doi:10.1364/OE.26.018664

15. Yoshida M, Yoshida K, Kasai K, Nakazawa M, et al. A 0.95ps, 10GHz, 60mW HCN Frequency-Stabilized and Mode-Locked Fiber Laser at $1.55 \mu \mathrm{m}$. San Jose: Lasers and Electro-optics (2016). doi:10.1364/CLEO_SI.2016.Stu1P.3

16. Bourne OL, Alcock AJ. Simplified Technique for Subnanosecond Pulse Generation and Injection Mode-Locking of a XeCl Laser. Appl Phys B (1985) 36(4):181-5. doi:10.1007/BF00704572
ZB: Supervision, Writing-Review and Editing. YL: Writing-Review and Editing. YY: Writing-Review and Editing. ZL: Supervision, Funding acquisition.

\section{FUNDING}

This work was supported by the National Natural Science Foundation of China (Nos. 62075056, 61927815, and 61905061).

17. Li X, Wang H, Qiao ZL, Guo X, Wang WJ, Ng GI, et al. Investigation of Regime Switching from Mode Locking to Q-Switching in a $2 \mu \mathrm{m} \mathrm{InGaSb/}$ AlGaAsSb Quantum Well Laser. Opt Express (2018) 26(7):8289. doi:10.1364/ OE.26.008289

18. Perego AM, Garbin B, Gustave F, Barland S, Prati F, de Valcárcel GJ. Coherent Master Equation for Laser Modelocking. Nat Commun (2020) 11(1):311. doi:10.1038/s41467-019-14013-4

19. Li Y, Tu W, Wang B, Zong N, Yang F, Zhang F, et al. High-Power NarrowLinewidth Q-Switched TEM00 Mode 355-nm Laser. IEEE Photon J. (2018) 10(3):1502509. doi:10.1109/JPHOT.2018.2825238

20. Zayhowski JJ, Dill C. Diode-pumped Passively Q-Switched Picosecond Microchip Lasers. Opt Lett (1994) 19(18):1427-9. doi:10.1364/OL.19.001427

21. Yang X, Zhang P, Xie W, Li L Double Q-Switch Ho:Sc $\mathrm{SiO}_{5}$ Laser by Acousto-Optic Modulator Combined with $\mathrm{Cr}^{2+}: \mathrm{ZnSe}$ Saturable Absorber. Opt Laser Technol (2018) 98:19-22. doi:10.1016/j.optlastec.2017.07.038

22. Cole B, Goldberg L, Hays AD. High-efficiency $2 \mu \mathrm{m}$ Tm:YAP Laser with a Compact Mechanical Q-Switch. Opt Lett (2018) 43(2):170-3. doi:10.1364/ OL.43.000170

23. Boyd RW, Rza̧ewski K, Narum P. Noise Initiation of Stimulated Brillouin Scattering. Phys Rev A (1990) 42(9):5514-21. doi:10.1103/PhysRevA.42.5514

24. Crofts GJ, Lamb RA, Damzen MJ. Experimental and Theoretical Investigation of Two-Cell Stimulated-Brillouin-Scattering Systems. J Opt Soc Am B (1991) 8(11):2282-8. doi:10.1364/JOSAB.8.002282

25. Wang HL, Cha S, Kong HJ, Wang YL, Lu ZW. Rotating Off-Centered Lens in SBS Phase Conjugation Mirror for High-Repetition-Rate Operation. Opt Express (2019) 27(7):9895-905. doi:10.1364/OE.27.009895

26. Tsubakimoto K, Yoshida H, Miyanaga N. High-average-power green Laser Using Nd:YAG Amplifier with Stimulated Brillouin Scattering PhaseConjugate Pulse-Cleaning Mirror. Opt Express (2016) 24(12):12557-64. doi:10.1364/OE.24.012557

27. Kang ZJ, Fan ZW, Huang YT, Zhang HB, Ge WQ, Li MS, et al. Highrepetition-rate, High-Pulse-Energy, and High-Beam-Quality Laser System Using an Ultraclean Closed-type SBS-PCM. Opt Express (2018) 26(6): 6560-71. doi:10.1364/OE.26.006560

28. Zhu Z-H, Chen P, Li H-W, Zhao B, Zhou Z-Y, Hu W, et al. Fragmentation of Twisted Light in Photon-Phonon Nonlinear Propagation. Appl Phys Lett (2018) 112(16):161103. doi:10.1063/1.5020082

29. Kong HJ, Park S, Cha S, Ahn H, Lee H, Oh J, et al. Conceptual Design of the Kumgang Laser: a High-Power Coherent Beam Combination Laser Using SC-SBS-PCMs towards a Dream Laser. High Pow Laser Sci Eng (2015) 3(1): 19-31. doi:10.1017/hpl.2014.49

30. Park S, Cha S, Oh J, Lee H, Ahn H, Churn KS, et al. Coherent Beam Combination Using Self-phase Locked Stimulated Brillouin Scattering Phase Conjugate Mirrors with a Rotating Wedge for High Power Laser Generation. Opt Express (2016) 24(8):8641-6. doi:10.1364/OE.24.008641

31. Cui C, Wang YL, Lu ZW, Yuan H, Wang Y, Chen Y, et al. Demonstration of $25 \mathrm{~J}, 10 \mathrm{~Hz}$, Nanosecond Laser Beam Combination System Based on Noncollinear Brillouin Amplification. Opt Express (2018) 26(25):32717-27. doi:10.1364/OE.26.03272710.1364/oe.26.032717

32. Hasi WLJ, Lu ZW, Gong S, Lin DY, He WM, Fan RQ. Investigation on the Output Energy Characteristic of Optical Limiting Based on Stimulated Brillouin Scattering. Appl Phys B (2008) 92:599-602. doi:10.1007/s00340-008-3115-2

33. Lu ZW, Lu YL, Yang J. Optical Limiting Effect Based on Stimulated Brillouin Scattering in $\mathrm{CCl}_{4}$. Chin Phys. (2003) 12(5):507-13. doi:10.1088/1009-1963/ $12 / 5 / 308$ 
34. Kang ZJ, Zhang HB, Yan XC, Lang Y, Bai ZA, Fan ZW. $200 \mathrm{~Hz}$ High Repetition Frequency SBS Pulse Width Compression experiment. Chin Opt (2018) 11(5):736-44. doi:10.3788/CO.20181105.0736

35. Wang H, Cha S, Wang Y, Kong HJ, Lu Z. SBS Pulse Compression Using Bulk Fused Silica by Diode-Pumped Solid-State Lasers at $1 \mathrm{kHz}$ Repetition Rate. Opt Laser Technol (2020) 128:106258. doi:10.1016/j.optlastec.2020.106258

36. Forbes A, de Oliveira M, Dennis MR. Structured Light. Nat Photon (2021) 15: 253-62. doi:10.1038/s41566-021-00780-4

37. Rubinsztein-Dunlop H, Forbes A, Berry MV, Dennis MR, Andrews DL, Mansuripur M, et al. Roadmap on Structured Light. J Opt (2017) 19:013001. doi:10.1088/2040-8978/19/1/013001

38. Courtial J, Dholakia K, Allen L, Padgett MJ. Second-harmonic Generation and the Conservation of Orbital Angular Momentum with High-Order Laguerre-Gaussian Modes. Phys Rev A (1997) 56:4193-6. doi:10.1103/ PhysRevA.56.4193

39. Wu H-J, Mao L-W, Yang Y-J, Rosales-Guzmán C, Gao W, Shi B-S, et al. Radial Modal Transitions of Laguerre-Gauss Modes during Parametric UpConversion: Towards the Full-Field Selection Rule of Spatial Modesfield Selection Rule of Spatial Modes. Phys Rev A (2020) 101:063805. doi:10.1103/ PhysRevA.101.063805

40. Mendonça JT, Thidé B, Then H. Stimulated Raman and Brillouin Backscattering of Collimated Beams Carrying Orbital Angular Momentum. Phys Rev Lett (2009) 102(18):185005. doi:10.1103/ PhysRevLett.102.185005

41. Gao W, Mu C, Li H, Yang Y, Zhu Z. Parametric Amplification of Orbital Angular Momentum Beams Based on Light-Acoustic Interaction. Appl Phys Lett (2015) 107(4):041119. doi:10.1063/1.4927699

42. Vieira J, Trines RMGM, Alves EP, Fonseca RA, Mendonça JT, Bingham R, et al. Amplification and Generation of Ultra-intense Twisted Laser Pulses via Stimulated Raman Scattering. Nat Commun (2016) 7(3):10371. doi:10.1038/ ncomms 10371

43. Zhu Z-H, Chen P, Sheng L-W, Wang Y-L, Hu W, Lu Y-Q, et al. Generation of strong Cylindrical Vector Pulses via Stimulated Brillouin Amplification. Appl Phys Lett (2017) 110(14):141104. doi:10.1063/1.4979588

44. Vieira J, Trines RMGM, Alves EP, Fonseca RA, Mendonça JT, Bingham R, et al. High Orbital Angular Momentum Harmonic Generation. Phys Rev Lett (2016) 117(26):265001. doi:10.1103/PhysRevLett.117.265001

45. Liu HX, Li YL, Gu XK, Hu WW, Zhang YP, Zhang YM. Research on Medium for Ultrashort Pulse Laser by SBS. Laser and Infrared (2019) 49(004):387-94. doi:10.3969/j.issn.1001-5078.2019.04.001

46. Bai Z, Yuan H, Liu Z, Xu P, Gao Q, Williams RJ, et al. Stimulated Brillouin Scattering Materials, Experimental Design and Applications: A Review. Opt Mater (2018) 75:626-45. doi:10.1016/j.optmat.2017.10.035

47. Maier M, Kaiser W, Giordmaine JA. Intense Light Bursts in the Stimulated Raman Effect. Phys Rev Lett (1966) 17(26):1275-7. doi:10.1103/ PhysRevLett.17.1275

48. Glass A. Design Considerations for Raman Lasers. IEEE J Quan Electron. (1967) 3(6):516-20. doi:10.1109/JQE.1967.1074552

49. Minck RW, Hagenlocker EE, Rado WG. Simultaneous Occurrence of and Competition between Stimulated Optical-Scattering Processes in Gases. J Appl Phys (1967) 38(5):2254-60. doi:10.1063/1.1709865

50. Culver WH, Vanderslice JTA, Townsend VWT. Controlled Generation of Intense Light Pulses in Reverse-pumped Raman Lasers. Appl Phys Lett (1968) 12(5):189-90. doi:10.1063/1.1651946

51. Rahn O, Maier M, Kaiser W. Stimulated Raman, Librational, and Brillouin Scattering in Water. Opt Commun (1969) 1(3):109-10. doi:10.1016/00304018(69)90022-4

52. Maier M, Kaiser W, Giordmaine JA. Backward Stimulated Raman Scattering. Phys Rev (1969) 177(2):580-99. doi:10.1103/PhysRev.177.580

53. Stolen RH, Ippen EP, Tynes AR. Raman Oscillation in Glass Optical Waveguide. Appl Phys Lett (1972) 20(2):62-4. doi:10.1063/1.1654046

54. Lowdermilk WH, Kachen GI. Stokes Pulse Growth in Transient Stimulated Raman Scattering. Appl Phys Lett (1975) 27(3):133-5. doi:10.1063/1.88381

55. Tan-No N, Shirahata T, Yokoto K, Inaba H. Coherent Transient Effect in Raman Pulse Propagation. Phys Rev A (1975) 12(1):159-68. doi:10.1103/ PhysRevA.12.159

56. Heuer A, Menzel R. Phase-conjugating Stimulated Brillouin Scattering Mirror for Low Powers and Reflectivities above $90 \%$ in an Internally
Tapered Optical Fiber. Opt Lett (1998) 23(11):834-6. doi:10.1364/ OL.23.000834

57. Ewing J, Haas R, Swingle J, George E, Krupke W. Optical Pulse Compressor Systems for Laser Fusion. IEEE J Quan Electron. (1979) 15(5):368-79. doi:10.1109/JQE.1979.1070010

58. Murray J, Goldhar J, Eimerl D, Szoke A. Raman Pulse Compression of Excimer Lasers for Application to Laser Fusion. IEEE J Quan Electron. (1979) 15(5):342-68. doi:10.1109/JQE.1979.1070009

59. Chiao RY, Townes CH, Stoicheff BP. Stimulated Brillouin Scattering and Coherent Generation of Intense Hypersonic Waves. Phys Rev Lett (1964) 12(21):592-5. doi:10.1103/PhysRevLett.12.592

60. Hon DT. Pulse Compression by Stimulated Brillouin Scattering. Opt Lett (1980) 5(12):516-8. doi:10.1364/OL.5.000516

61. Yuan H, Wang Y, Lu Z, Liu Z, Bai Z, Liu R, et al. SRS Characteristics and its Influence on SBS Pulse Compression in a Fluorocarbon Liquid. Laser Part Beams (2017) 35(1):114-9. doi:10.1017/S026303461600094X

62. Damzen M, Hutchinson H. Laser Pulse Compression by Stimulated Brillouin Scattering in Tapered Waveguides. IEEE J Quan Electron. (1983) 19(1):7-14. doi:10.1109/JQE.1983.1071718

63. Zhao Z, Dong Y, Pan S, Liu C, Chen J, Tong L, et al. Performance of Large Aperture Tapered Fiber Phase Conjugate Mirror with High Pulse Energy and 1-kHz Repetition Rate. Opt Express (2012) 20(2):1896-902. doi:10.1364/OE.20.001896

64. Damzen MJ, Hutchinson MHR. High-efficiency Laser-Pulse Compression by Stimulated Brillouin Scattering. Opt Lett (1983) 8(6):313-5. doi:10.1364/ OL.8.000313

65. Gorbunov VA, Papernyı̆ SB, Petrov VF, Startsev VR. Time Compression of Pulses in the Course of Stimulated Brillouin Scattering in Gases. Sov J Quan Electron. (1983) 13(7):900-5. doi:10.1070/QE1983v013n07ABEH004367

66. Tomov IV, Fedosejevs R, Mcken DCD. High-efficiency Stimulated Brillouin Scattering of $\mathrm{KrF}$ Laser Radiation in $\mathrm{SF}_{6}$. Opt Lett (1984) 9(9):405-7. doi:10.1364/OL.9.000405

67. Tomov I, Fedosejevs R, Mcken D. Stimulated Brillouin Scattering of KrF Laser Radiation in Dichlorodifluoromethane. IEEE J Quan Electron. (1985) 21(1):9-11. doi:10.1109/JQE.1985.1072533

68. Buzyalis RR, Dementjev AS, Kosenko EK. Formation of Subnanosecond Pulses by Stimulated Brillouin Scattering of Radiation from a Pulse-Periodic YAG:Nd Laser. Sov J Quan Electron. (1985) 15(10):1335-7. doi:10.1070/ QE1985v015n10ABEH007759

69. Davydov MA, Shipilov KF, Shmaonov TA. Formation of Highly Compressed Stimulated Brillouin Scattering Pulses in Liquids. Sov J Quan Electron. (1986) 16(10):1402-3. doi:10.1070/QE1986v016n10ABEH007597

70. Gă̌zhauskas É, Krushas V, Nedbaev NY, Petrenko RA, Piskarskas A, Smil'gyavichyus V. Generation of Picosecond Pulses as a Result of Stimulated Brillouin Scattering in Liquids. Sov J Quan Electron. (1986) 16(6):854-5. doi:10.1070/QE1986v016n06ABEH006956

71. Kmetik V, Fiedorowicz H, Andreev AA, Witte KJ, Daido H, Fujita H, et al. Reliable Stimulated Brillouin Scattering Compression of Nd:YAG Laser Pulses with Liquid Fluorocarbon for Long-Time Operation at $10 \mathrm{~Hz}$. Appl Opt (1998) 37(30):7085-90. doi:10.1364/AO.37.007085

72. Yoshida H, Fujita H, Nakatsuka M, Fujinoki A. Temporal Compression by Stimulated Brillouin Scattering of Q-Switched Pulse with Fused Quartz Glass. Jpn J Appl Phys (2004) 43(8B):L1103-L1105. doi:10.1143/JJAP.43.L1103

73. Yoshida H, Fujita $H$, Nakatsuka M, Ueda T, Fujinoki A. Temporal Compression by Stimulated Brillouin Scattering of Q-Switched Pulse with Fused-Quartz and Fused-Silica Glass from $1064 \mathrm{~nm}$ to $266 \mathrm{~nm}$ Wavelength. Laser Part Beams (2007) 25(03):481-8. doi:10.1017/S0263034607000596

74. Liu Z, Wang Y, Wang Y, Li S, Bai Z, Lin D, et al. Pulse-shape Dependence of Stimulated Brillouin Scattering Pulse Compression to Sub-phonon Lifetime. Opt Express (2018) 26(5):5701-10. doi:10.1364/OE.26.005701

75. Bai Z, Wang Y, Lu Z, Yuan H, Jiang L, Tan T, et al. Efficient KDP Frequency Doubling SBS Pulse Compressed 532 nm Hundred Picosecond Laser. Optik (2016) 127(20):9201-5. doi:10.1016/j.ijleo.2016.07.021

76. Liu Z, Wang Y, Bai Z, Wang Y, Jin D, Wang H, et al. Pulse Compression to One-Tenth of Phonon Lifetime Using Quasi-Steady-State Stimulated Brillouin Scattering. Opt Express (2018) 26(18):23051-60. doi:10.1364/ OE.26.023051

77. Gă̌zhauskas É, Piskarskas A, Smil'gyavichyus V, Stalyunas K. Space and Time Structure of Ultrashort Pulses Formed by Opposed Stimulated 
Scattering of Laser Beams. Sov J Quan Electron. (1987) 17(5):650-3. doi:10.1070/QE1987v017n05ABEH009067

78. Schiemann S, Ubachs W, Hogervorst W. Efficient Temporal Compression of Coherent Nanosecond Pulses in a Compact SBS Generator-Amplifier Setup. IEEE J Quan Electron. (1997) 33(3):358-66. doi:10.1109/3.556004

79. Wang Y., Lu Z, Guo Q, Wu P, Zheng Z, He W A New Circulating Two-Cell Structure for Stimulated Brillouin Scattering Phase Conjugation Mirrors with 1-J Load and 10-Hz Repetition Rate. Chin Opt Lett (2010) 8(11):1064-6. doi:10.3788/COL20100811.1064

80. Zheng ZX, Hasi WLJ, Zhao H, Cheng SX, Wang XY, Lin DY, et al. Compression Characteristics of Two New SBS Mediums to Generate 100ps Pulse for Shock Ignition. Appl Phys B (2014) 116(3):659-63. doi:10.1007/ s00340-013-5749-y

81. Hasi WLJ, Qiao Z, Cheng SX, Wang XY, Zhong ZM, Zheng ZX, et al. Characteristics of SBS Hundreds Picosecond Pulse Compression and Influence of Energy on Pulse Stability in FC-770. Opt Commun (2013) 311:375-9. doi:10.1016/j.optcom.2013.05.056

82. Ogino J, Miyamoto S, Matsuyama T, Sueda K, Yoshida H, Tsubakimoto K, et al. Two-stage Optical Parametric Chirped-Pulse Amplifier Using Subnanosecond Pump Pulse Generated by Stimulated Brillouin Scattering Compression. Appl Phys Express (2014) 7(12):122702. doi:10.7567/ APEX.7.122702

83. Yoshida H, Fujita H, Nakatsuka M, Ueda T, Fujinoki A. Compact TemporalPulse-Compressor Used in Fused-Silica Glass at $1064 \mathrm{~nm}$ Wavelength. Jpn J Appl Phys (2007) 46(3):L80-L82. doi:10.1143/JJAP.46.L80

84. Yoshida H, Hatae T, Fujita H, Nakatsuka M, Kitamura S. A High-Energy 160ps Pulse Generation by Stimulated Brillouin Scattering from Heavy Fluorocarbon Liquid at $1064 \mathrm{~nm}$ Wavelength. Opt Express (2009) 17(16): 13654-62. doi:10.1364/OE.17.013654

85. Daito I, Kando M, Kotaki H, Hayashi Y, Kizugawa J, Shizuma T, et al. High Flux Laser-Compton Scattered Gamma-Ray Source by Compressed Nd:YAG Laser Pulse. New Orleans: International Particle Accelerator Conference (2012).

86. Zhu X, Lu Z, Wang Y. High Stability, Single Frequency, 300 mJ, 130 Ps Laser Pulse Generation Based on Stimulated Brillouin Scattering Pulse Compression. Laser Part Beams (2015) 33(01):11-5. doi:10.1017/ S0263034614000597

87. Wang Y, Liu Z, Yuan H, Bai Z, Wang H, Zhu X, et al. A Promotion of Stability for Temporal Compression Based on SBS in an Interferometric Scheme. J Mod Opt (2016) 63(17):1734-40. doi:10.1080/09500340.2016.1171406

88. Bai Z, Wang Y, Lu Z, Yuan H, Zheng Z, Li S, et al. High Compact, High Quality Single Longitudinal Mode Hundred Picoseconds Laser Based on Stimulated Brillouin Scattering Pulse Compression. Appl Sci (2016) 6(1):29. doi:10.3390/app6010029

89. Bai Z, Wang Y, Lu Z, Jiang L, Yuan H, Liu Z. Demonstration of an Ultraviolet Stimulated Brillouin Scattering Pulse Compressed Hundred Picosecond Laser in LiB3O5crystals. J Opt (2017) 19(8):085502. doi:10.1088/2040-8986/aa74d6

90. Wang H, Cha S, Kong HJ, Wang Y, Lu Z. Sub-nanosecond Stimulated Brillouin Scattering Pulse Compression Using HT270 for kHz Repetition Rate Operation. Opt Express (2019) 27(21):29789-802. doi:10.1364/ OE.27.029789

91. Mitra A, Yoshida H, Fujita H, Nakatsuka M. Sub Nanosecond Pulse Generation by Stimulated Brillouin Scattering Using FC-75 in an Integrated Setup with Laser Energy up to 1.5 J. Jpn J Appl Phys (2006) 45(3A):1607-11. doi:10.1143/JJAP.45.1607

92. Kmetik V, Yoshida H, Fujita H, Nakatsuka M, Yamanaka T. Very High Energy SBS Phase Conjugation and Pulse Compression in Fluorocarbon Liquids. Proc SPIE-The Int Soc Opt Eng (2000) 3889:818-26. doi:10.1117/12.380865

93. Fedosejevs R, Tomov IV, Mcken DCD, Offenberger AA. Experimental Study of an SF6Brillouin Amplifier Pumped by KrF Laser Radiation. Appl Phys Lett (1984) 45(4):340-2. doi:10.1063/1.95262

94. Fedosejevs R, Offenberger A. Subnanosecond Pulses from a KrF Laser Pumped SF6Brillouin Amplifier. IEEE J Quan Electron. (1985) 21(10): 1558-62. doi:10.1109/JQE.1985.1072567

95. Dane CB, Neuman WA, Hackel LA. High-energy SBS Pulse Compression. IEEE J Quan Electron. (1994) 30(8):1907-15. doi:10.1109/3.301654

96. Lancia L, Marquès J-R, Nakatsutsumi M, Riconda C, Weber S, Hüller S, et al. Experimental Evidence of Short Light Pulse Amplification Using Strong-
Coupling Stimulated Brillouin Scattering in the Pump Depletion Regime. Phys Rev Lett (2010) 104(2):025001. doi:10.1103/PhysRevLett.104.025001

97. Feng C, Xu X, Diels J-C. Generation of 300 ps Laser Pulse with 12 J Energy by Stimulated Brillouin Scattering in Water at $532 \mathrm{~nm}$. Opt Lett (2014) 39(12): 3367-70. doi:10.1364/OL.39.003367

98. Xu X, Feng C, Diels J-C. Optimizing Sub-ns Pulse Compression for High Energy Application. Opt Express (2014) 22(11):13904-15. doi:10.1364/ OE.22.013904

99. Feng $\mathrm{C}, \mathrm{Xu} \mathrm{X}$, Diels J-C. High-energy Sub-phonon Lifetime Pulse Compression by Stimulated Brillouin Scattering in Liquids. Opt Express (2017) 25(11):12421-34. doi:10.1364/OE.25.012421

100. Takahashi E, Kuwahara K, Matsumoto Y, Owadano Y. High-intensity Short $\mathrm{KrF}$ Laser-Pulse Generation by Saturated Amplification of Truncated Leading-Edge Pulse. Opt Commun (2000) 185(4-6):431-7. doi:10.1016/ S0030-4018(00)01042-7

101. Kuwahara K, Takahashi E, Matsumoto Y, Kato S, Owadano Y, Kato S, et al. Short-pulse Generation by Saturated KrF Laser Amplification of a Steep Stokes Pulse Produced by Two-step Stimulated Brillouin Scattering. J Opt Soc Am B (2000) 17(11):1943-7. doi:10.1364/JOSAB.17.001943

102. Wang $\mathrm{C}, \mathrm{Lu} \mathrm{ZW}, \mathrm{He}$ WM. Picosecond Pulse Generation by Stimulated Brillouin Scatte-Ring Compressor. Powerful Laser Part Beam (2003) 15(012): 1184-6. doi:10.1023/A:1022289509702

103. Wang C, Lu ZW, Lin DY, He WM. Laser Pulse with Duration Shorter Than 1/ 4 of Hypersound Oscillation Period of Medium Achieved by Stimulated Brillouin Compressor. Chin J Lasers (2006) 33:226-8.

104. Marcus G, Pearl S, Pasmanik G. Stimulated Brillouin Scattering Pulse Compression to 175 ps in a Fused Quartz at $1064 \mathrm{~nm}$. J Appl Phys (2008) 103(10):103105. doi:10.1063/1.2931001

105. Hasi WLJ, Zhao H, Lin DY, He WM, Lu ZW. Characteristics of Perfluorinated Amine Media for Stimulated Brillouin Scattering in Hundreds of Picoseconds Pulse Compression at $532 \mathrm{~nm}$. Chin Opt Lett (2015) 13: 061901-5. English version. doi:10.3788/COL201513.061901

106. Pivinskii EG, Akulinichev VV, Gorbunov VA. Nd:YAG Laser Pulse Compression by Three-Stage Stimulated Brillouin and Raman Scatterings. Proc SPIE Int Soc Opt Eng (1997) 2986:231-8. doi:10.1117/12.270002

107. Liu Z, Wang Y, Wang H, Bai Z, Li S, Zhang H, et al. Pulse Temporal Compression by Two-Stage Stimulated Brillouin Scattering and LaserInduced Breakdown. Appl Phys Lett (2017) 110(24):241108. doi:10.1063/ 1.4986437

108. Neshev D, Velchev I, Majewski WA, Hogervorst W, Ubachs W. SBS Pulse Compression to 200 ps in a Compact Single-Cell Setup. Appl Phys B: Lasers Opt (1999) 68(4):671-5. doi:10.1007/s00340990145410.1007/s003400050684

109. Wang Y, Zhu X, Lu Z, Zhang H. Generation of 360 ps Laser Pulse with 3 J Energy by Stimulated Brillouin Scattering with a Nonfocusing Scheme. Opt Express (2015) 23(18):23318-28. doi:10.1364/OE.23.023318

110. Andreev AA, Sutyagin AN. Feasibility of Optical Pulse Compression by Stimulated Brillouin Scattering in a Plasma. Sov J Quan Electron. (1989) 19(12):1579-82. doi:10.1070/QE1989v019n12ABEH009826

111. Andreev AA, Riconda C, Weber S. Generation and Amplification of Ultrashort Light Pulses in the Strongly Coupled Regime of SBS in Plasma. Phys Plasmas (2005) 41(3):414-7. doi:10.1021/je9501853

112. Andreev AA, Riconda C, Tikhonchuk VT, Weber S. Short Light Pulse Amplification and Compression by Stimulated Brillouin Scattering in Plasmas in the Strong Coupling Regime. Phys Plasmas (2006) 13(5): 053110. doi:10.1063/1.2201896

113. Weber S, Riconda C, Lancia L, Marquès J-R, Mourou GA, Fuchs J. Amplification of Ultrashort Laser Pulses by Brillouin Backscattering in Plasmas. Phys Rev Lett (2013) 111(5):055004. doi:10.1103/ PhysRevLett.111.055004

114. Peng H, Wu ZH, Zuo YL, Zhang ZM, Zhou KN, Su JQ. Single Laser Pulse Compression via Strongly Coupled Stimulated Brillouin Scattering in Plasma. Phys Plasmas (2016) 23(7):073516. doi:10.1063/1.4959173

115. Buono WT, Santos A, Maia MR, Pereira LJ, Tasca DS, Dechoum K, et al. Chiral Relations and Radial-Angular Coupling in Nonlinear Interactions of Optical Vortices. Phys Rev A (2020) 101:043821. doi:10.1103/ PhysRevA.101.043821

116. Wu H-J, Yang H-R, Rosales-Guzmán C, Gao W, Shi B-S, Zhu Z-H. Vectorial Nonlinear Optics: Type-II Second-Harmonic Generation Driven by Spin- 
Orbit-Coupled Fieldsfields. Phys Rev A (2019) 100:053840. doi:10.1103/ PhysRevA.100.053840

117. R Mildren J Rabeau, editors. Optical Engineering of Diamond. John Wiley \& Sons (2013).

118. Bai Z, Williams RJ, Kitzler O, Sarang S, Spence DJ, Wang Y, et al. Diamond Brillouin Laser in the Visible. APL Photon (2020) 5(3):031301. doi:10.1063/ 1.5134907

119. Bai Z, Zhang Z, Wang K, Gao J, Zhang Z, Yang X, et al. Comprehensive Thermal Analysis of Diamond in a High-Power Raman Cavity Based on FVM-FEM Coupled Method. Nanomaterials (2021) 11(6):1572. doi:10.3390/nano11061572

120. Williams RJ, Kitzler O, Bai Z, Sarang S, Jasbeer H, McKay A, et al. High Power diamond Raman Lasers. IEEE J Select Top Quan Electron. (2018) 24(5): 1602214. doi:10.1109/JSTQE.2018.2827658

121. Pessot M, Maine P, Mourou G. 1000 Times Expansion/compression of Optical Pulses for Chirped Pulse Amplification. Opt Commun (1987) 62(6):419-21. doi:10.1016/0030-4018(87)90011-3

122. Pessot M, Harter DJ, Squier J, Mourou G. Chirped-pulse Amplification of 100-fsec Pulses. Opt Lett (1989) 14(15):797-9. doi:10.1364/OL.14.000797

123. Perry MD, Mourou G. Terawatt to Petawatt Subpicosecond Lasers. Science (1994) 264(5161):917-24. doi:10.1126/science.264.5161.917

124. Morin P, Dubertrand J, Beaure d'Augeres P, Quiquempois Y, Bouwmans G, Mussot A, et al. $\mu$ L Level Raman-Assisted Fiber Optical Parametric Chirped-Pulse Amplification. Opt Lett (2018) 43(19):4683-86. doi:10.1364/OL.43.004683

125. Guo Z, Yu L, Wang J, Wang C, Liu Y, Gan Z, et al. Improvement of the Focusing Ability by Double Deformable Mirrors for 10-PW-level Ti: Sapphire Chirped Pulse Amplification Laser System. Opt Express (2018) 26(20): 26776-86. doi:10.1364/OE.26.026776

126. Zhu Z, Gao W, Mu C, Li H. Reversible Orbital Angular Momentum PhotonPhonon Conversion. Optica (2016) 3(2):212-7. doi:10.1364/ OPTICA.3.000212
127. Lombardi GG. Pulse Compression by Stimulated Brillouin Scattering. Boston: O-E/Fiber Laser (1989). doi:10.1364/OL.5.000516

128. Offenberger AA, Thompson DC, Fedosejevs R, Harwood B, Santiago J, Manjunath HR. Experimental and Modeling Studies of a Brillouin Amplifier. IEEE J Quan Electron. (1993) 29(1):207-16. doi:10.1109/3.199261

129. Yuan H, Wang YL, Lu ZW, Zheng ZX. Small-Scale Self-Focusing of 200 ps Laser Pulses in Brillouin Amplification. Chin Phys. B (2015) 24(009): 094210-270. doi:10.1088/1674-1056/24/9/094210

130. Yuan H, Lu ZW, Wang YL, Zheng ZX, Chen Y. Hundred Picoseconds Laser Pulse Amplification Based on Scalable Two-Cells Brillouin Amplifier. Laser Part Beams (2014) 32(03):369-74. doi:10.1017/ S0263034614000238

Conflict of Interest: The authors declare that the research was conducted in the absence of any commercial or financial relationships that could be construed as a potential conflict of interest.

Publisher's Note: All claims expressed in this article are solely those of the authors and do not necessarily represent those of their affiliated organizations, or those of the publisher, the editors and the reviewers. Any product that may be evaluated in this article, or claim that may be made by its manufacturer, is not guaranteed or endorsed by the publisher.

Copyright $\odot 2021 \mathrm{Cao}$, Wang, Bai, Li, Yu and Lu. This is an open-access article distributed under the terms of the Creative Commons Attribution License (CC BY). The use, distribution or reproduction in other forums is permitted, provided the original author(s) and the copyright owner(s) are credited and that the original publication in this journal is cited, in accordance with accepted academic practice. No use, distribution or reproduction is permitted which does not comply with these terms. 